\title{
The effect of crystallinity on the mechanical properties of plain woven carbon reinforced composites using polypropylene
}

\author{
H. Katogi \& K. Takemura \\ Department of Mechanical Engineering, Kanagawa University, Japan
}

\begin{abstract}
In this study, the effect of crystallinity on the mechanical properties of carbon fiber reinforced thermoplastics (CFRTP) was investigated. Plain woven carbon fiber was used as reinforcement. Polypropylene (PP) and maleic anhydride modified polypropylene (MAPP) were used as a matrix. The crystallinity of PP was controlled by using heat treatment after hot press molding of CFRTP. The range of crystallinity of PP and MAPP were from $26 \%$ to $40 \%$. Flexural and izod impact tests of CFRTP were conducted based on Japanese Industrial Standard (JIS) K 7074 and K 7110, respectively. And double-notched test of CFRTP was conducted. Crystal morphologies of single carbon fiber added thermoplastics were observed by using polarization microscope. As a result, flexural strength and izod impact the value of CFRTP using PP increased with an increase of crystallinity. Flexural strength and izod impact value of CFRTP using MAPP almost did not change with an increase of crystallinity. But interfacial shear strength of CFRTP using MAPP increased with an increase of crystallinity. Crystallizations of PP and MAPP occurred around carbon fiber after heat treatment. Therefore, interfacial adhesion between fiber and resin increased with an increase of crystallinity.

Keywords: carbon fiber, PP, MAPP, flexural property, interlaminar shear strength, crystallinity.
\end{abstract}

\section{Introduction}

Carbon fiber reinforced plastics has high specific strength and stuffiness. From viewpoints of moldability and recycle for automobile, carbon fiber reinforced thermoplastics (CFRTP) using thermoplastic as matrix has been focused. 
Polypropylene (PP) as thermoplastics has high moldability and thermal stability. The specific gravity of PP is low. So when PP is used as matrix, weight reduction of industrial product can be achieved. CFRTP using PP has been used for automobile materials such as instrument panel, door module and body [1, 2]. Mechanical properties of CFRTP using PP should be investigated for various application of automobile parts.

PP is crystalline resin and its molecular chain is arranged regularly. So, mechanical properties of PP depend on crystallinity. Okada and Hikosaka [3] reported that tensile property of PP increased with an increase of crystallinity. Interfacial adhesion between fiber and matrix is very low when PP is used as matrix. Many reports [4-9] of interfacial adhesion of composite using PP have been published. Generally, interfacial adhesion between fiber and matrix was greatly improved by using maleic anhydride modified polypropylene (MAPP) as matrix. But, few papers have been published about the effect of crystallinity on mechanical properties of CFRTP using MAPP and PP.

In this study, effects of crystallinity on mechanical properties of carbon fiber reinforced PP and MAPP were investigated.

\section{Specimen and testing method}

\subsection{Materials}

Plain woven carbon fiber (Torayca cloth CO6343, Toray Co., Ltd.) was used as reinforcement. Polypropylene (PP-N-AN, Shin-Kobe Electric Machinery Co., Ltd.) and maleic anhydride modified polypropylene (Umex1010, Sanyo Chemical Industries Co., Ltd.) are used for maleic modified matrix. The maleic anhydride modification density is about $5 \%$. As the molding condition of the composite using PP as matrix, molding pressure was $1.8 \mathrm{MPa}$, temperature was $190^{\circ} \mathrm{C}$ and time was $30 \mathrm{~min}$. Prior to molding of the composite using MAPP as matrix, a prepreg sheets were made using CF and MAPP. Composite using prepreg sheets were molded by hot press method. Molding pressure was $0.6 \mathrm{MPa}$, temperature was $190^{\circ} \mathrm{C}$ an time was $15 \mathrm{~min}$. Fiber volume fractions of the specimens were $40 \%$. In this study, carbon fiber reinforced PP and MAPP are named $\mathrm{CF} / \mathrm{PP}$ and $\mathrm{CF} / \mathrm{MAPP}$, respectively.

\subsection{Geometry and dimensions of specimen}

Static three point flexural test was conducted based on JIS K 7074. The specimen size was $100 \mathrm{~mm}$ length, $15 \mathrm{~mm}$ wide and $2 \mathrm{~mm}$ thickness. Izod impact test was conducted based on JIS K 7110. The specimen size was $80 \mathrm{~mm}$ length, $10 \mathrm{~mm}$ wide and $4 \mathrm{~mm}$ thickness. Notching length was $2 \mathrm{~mm}$. Double-notched test was conducted. The Specimen size was $25 \mathrm{~mm}$ length, $10 \mathrm{~mm}$ wide and $3.5 \mathrm{~mm}$ thickness. Notching length was $1.75 \mathrm{~mm}$. The schematic drawing of the specimen for double-notched test is shown in figure 1 . 


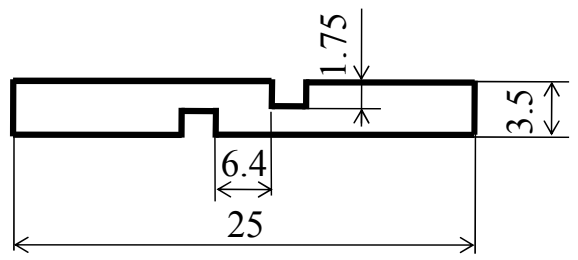

Figure 1: Schematic drawing of specimen for double notched test.

\subsection{Control of cristallinity of specimen}

Heat treatment was conducted to control crystallinity of specimens. The environmental temperature was $120^{\circ} \mathrm{C}$ and heating time was from 2 to $12 \mathrm{hrs}$.

After static three point flexural and izod impact tests, differential scanning calorimetry (DSC) test was conducted based on JIS K 7121. Crystallinity of specimen was calculated by following equation (1).

$$
X c(\%)=\frac{\Delta H_{m}}{\Delta H_{p p}} \times 100
$$

where $X \mathrm{c}$ is crystallinity, $\Delta H_{\mathrm{m}}$ is heat of fusion and $\Delta H_{\mathrm{pp}}$ is heat of fusion of a perfect crystal material. The ranges of crystallinity of PP and MAPP were from $26 \%$ to $40 \%$.

\subsection{Static three point flexural testing method}

Static flexural test was conducted by using universal testing machine (Shimadzu Co., Ltd., Autograph (AG-IS)). The static three point flexural test was referred to JIS K 7074. The crosshead speed was $5 \mathrm{~mm} / \mathrm{min}$. The gauge length was $80 \mathrm{~mm}$.

\subsection{Izod impact testing method}

Izod impact test was conducted by using shock machine (Toyo seiki seisaku-sho, Co., Ltd., Digital impact tester (DG-IB)). The izod impact test was referred to JIS K 7110. Impact direction was edgewise.

\subsection{Double-notched testing method}

Double-notched test of CFRTP were conducted by using universal testing machine. The crosshead speed was $1 \mathrm{~mm} / \mathrm{min}$. The gauge length was $18 \mathrm{~mm}$. Inter laminar shear strength of CFRTP was calculated by following equation (2).

$$
\tau=\frac{3 P}{4 b h}
$$

where $P$ is force, $b$ is width and $h$ is thickness. 


\subsection{Crystal and macroscopic observation}

Crystal morphologies of single carbon fiber added thermoplastics were observed by using polarization microscope (OLYMPUS Co., Ltd., POS). Fracture morphologies of CFRTP were observed by using optical microscope (OLYMPUS Co., Ltd., SZX7) after izod impact test.

\section{Results and discussion}

\subsection{Static three point flexural test}

Figure 2 shows the relationship between flexural properties and crystallinity. In case that crystallinity was $40 \%$, flexural strength of CF/MAPP was $90 \%$ higher than that of CF/PP and flexural modulus of CF/MAPP was 92\% higher. Their results implied that interfacial adhesion between fiber and resin was improved by maleic anhydride modification [9].

Flexural strength and modulus of CF/PP increased linearly with an increase of crystallinity of PP. Flexural strength of CF/MAPP slightly decreased with an increase of crystallinity of MAPP, and the flexural modulus almost did not change. Therefore, crystallinity was effective on the flexural strength and modulus of the composites using PP. But crystallinity of the composite using MAPP as matrix was not effective on flexural strength and modulus of composite. This is because the strength and modulus CF/MAPP was already improved by MAPP.

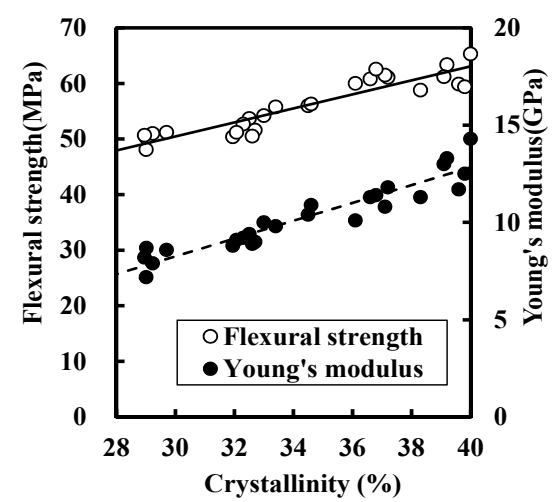

(a)

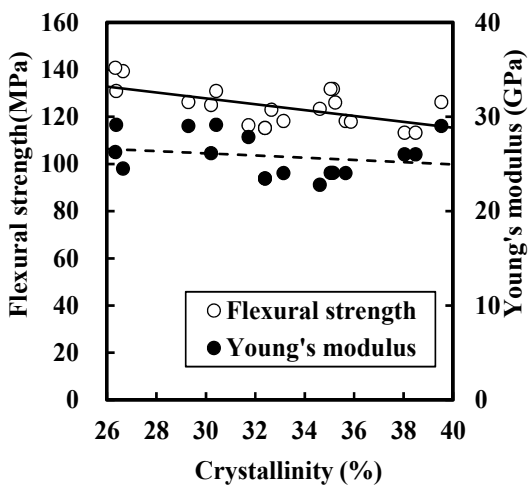

(b)

Figure 2: Relationship between flexural property and crystallinity. (a) CF/PP, (b) CF/MAPP.

Figure 3 shows stress - strain curves of CF/MAPP. Knee points on stress strain curves of CF/MAPP were found. In case of crystallinity $32 \%$, stress at knee point of CF/MAPP was $82 \mathrm{MPa}$. In case of crystallinity $38 \%$, stress at knee 
point of CF/MAPP was $104 \mathrm{MPa}$. Their results implied that interfacial adhesion of CF/MAPP increased with an increase of crystallinity. Therefore, crack initiation of CF/MAPP was prevented by an improvement of stress at knee point.

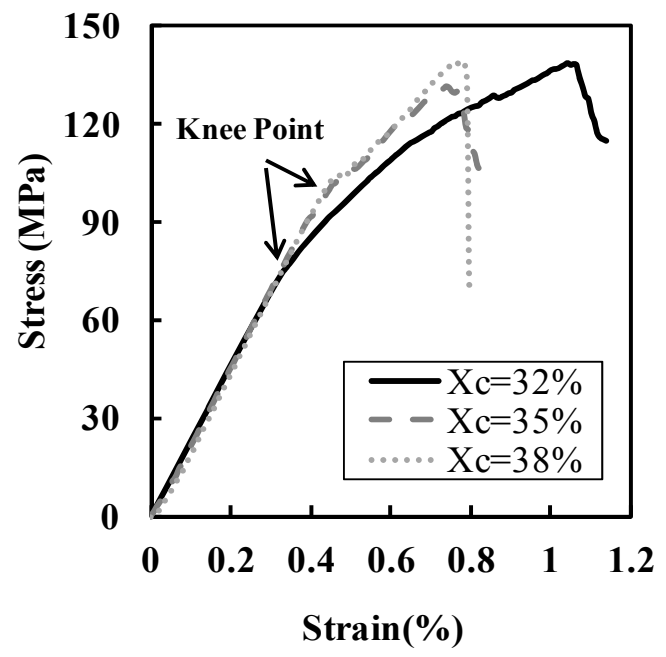

Figure 3: Stress - strain curves of CF/MAPP.

\subsection{Polarization microscope observation}

Figure 4 shows polarization microscopic of CF/PP. Figure 5 shows polarization microscopic observation of CF/MAPP. From figures 4 and 5, a white region in PP and MAPP was found. This white region in PP and MAPP increased by heat treatment. So, it is understood that crystallizations in PP and MAPP were grown by heat treatment. Crystallizations in crystalline polymer grow around carbon fiber $[10,11]$. Therefore, it is understood that crystallizations in PP and MAPP grew around carbon fiber after heat treatment on this experiment.

\subsection{Izod impact test}

Figure 6 shows the relationship between izod impact value and crystallinity. In case of crystallinity $38 \%$, izod impact value of CF/MAPP was $45 \%$ lower than that of $\mathrm{CF} / \mathrm{PP}$. Izod impact value of $\mathrm{CF} / \mathrm{PP}$ increased with an increase of crystallinity, but that of CF/MAPP almost did not change.

Figure 7 shows fracture morphology of CF/PP and CF/MAPP after izod impact tests. From figure 7 (a), PP as matrix was failed, but a part of fiber was not failed. From figure 7 (b), the brittle fracture of CF/MAPP probably occurred by brittleness of MAPP as matrix. In fracture morphology of CF/MAPP, all carbon fibers were failed, and pull-outs of carbon fibers were not found. Therefore, interfacial adhesion between fiber and resin was improved by maleic anhydride modification, so brittle fracture of CF/MAPP occurred. 


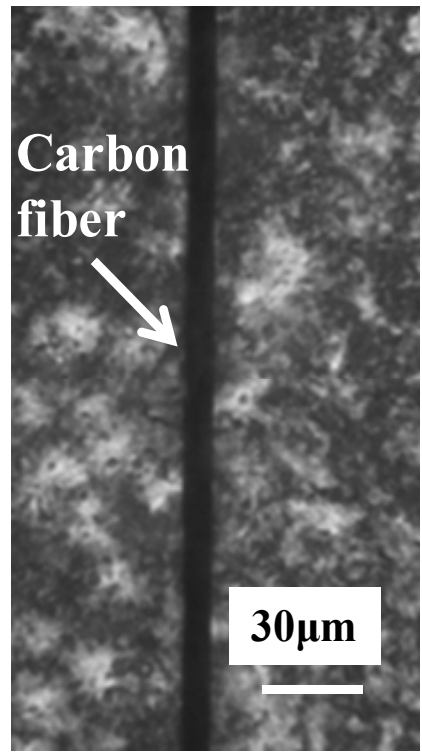

(a)

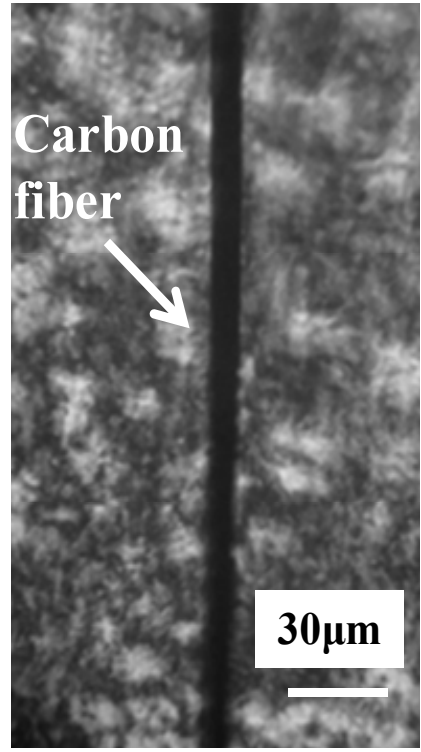

(b)

Figure 4: Polarization microscope of CF/PP. (a) Non-treatment, (b) heat treatment.

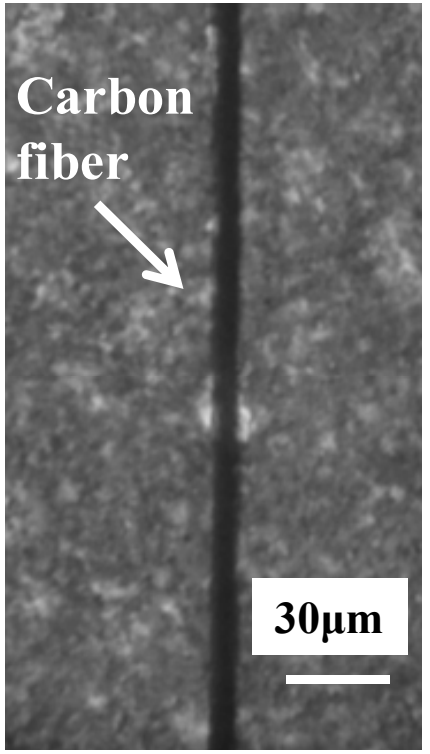

(a)

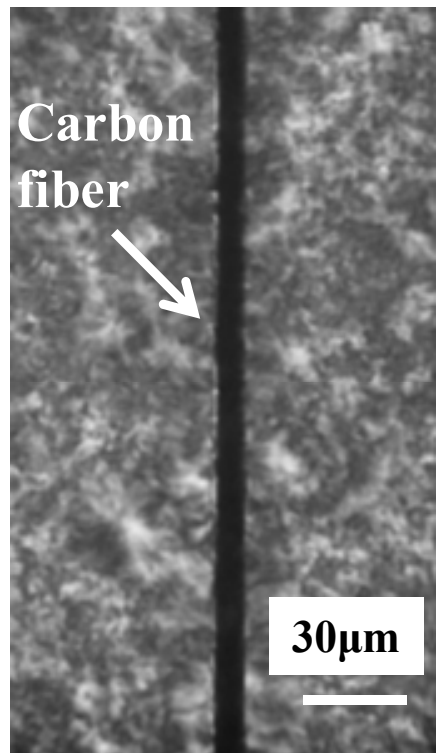

(b)

Figure 5: Polarization microscopic observation of CF/MAPP. (a) Nontreatment, (b) heat treatment. 


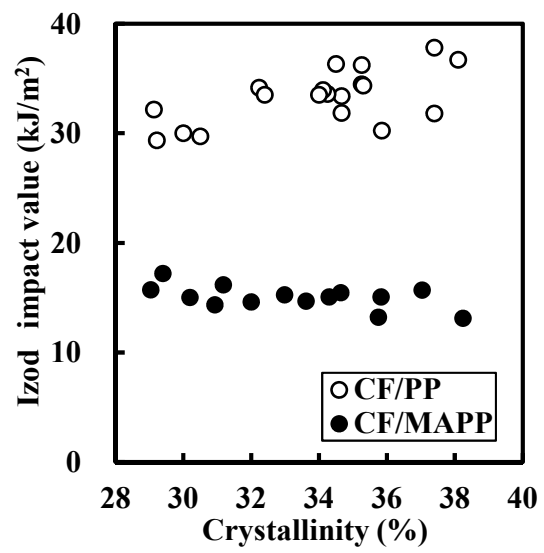

Figure 6: Relationship between izod impact value and crystallinity.

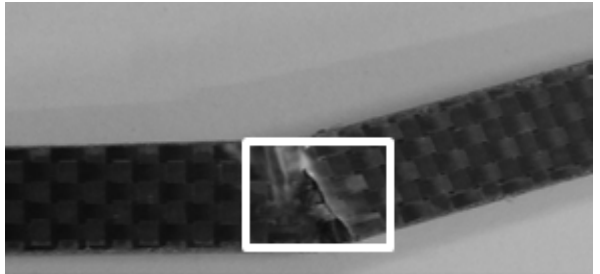

(a)

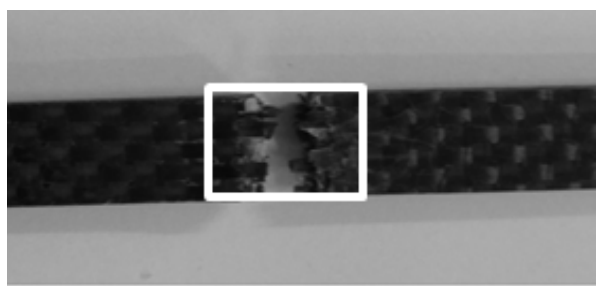

(b)
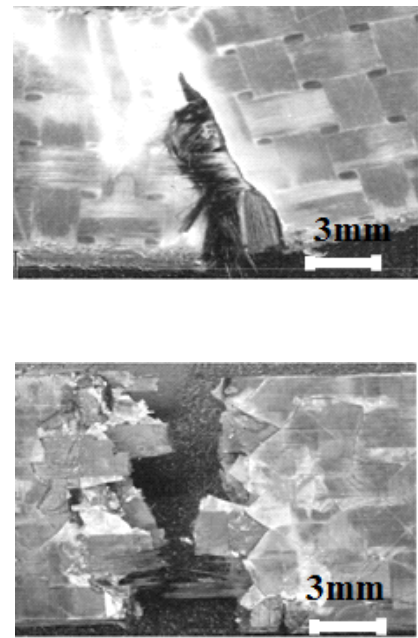

(a) $\mathrm{CF} / \mathrm{PP}$, (b) CF/MAPP.

\subsection{Double-notched test}

From the results in session 3.1, stress at knee point of CF/MAPP was improved by using maleic anhydride modification. So, interlaminar shear strength of CF/MAPP was investigated by using double-notched test. Figure 8 shows the relationship between interlaminar shear strength and crystallinity. Interlaminar shear strength of CF/MAPP increased with an increase of crystallinity of MAPP. Interfacial adhesion between fiber and resin was increased by an increase of crystallinity of MAPP. Therefore it is understood that interlaminar shear strength of CF/MAPP increased based on the crystallinity. 


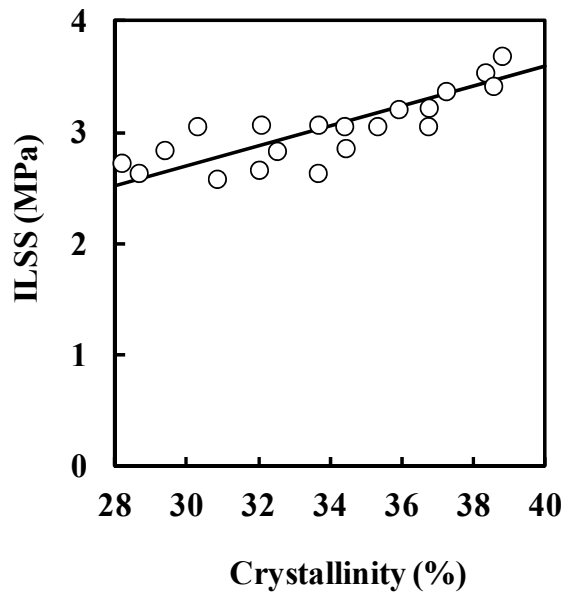

Figure 8: Relationship between interlaminar shear strength and crystallinity.

\section{Conclusions}

In this study, the effect of crystallinity on the mechanical properties of carbon fiber reinforced thermoplastics was investigated.

(1) Flexural strength and modulus of the composite using polypropylene increased with an increase of crystallinity of polypropylene. But an increase of crystallinity of maleic anhydride modified polypropylene was not effective in the improvement of strength and modulus of the composite.

(2) In case of izod impact test, the brittle fracture of composite occurred when maleic anhydride modified polypropylene was used as matrix. So, the izod impact value of the composite using maleic anhydride modified polypropylene was lower than that of composite using unmodified polypropylene. When the range of crystallinity of matrix was from $28 \%$ to $40 \%$, izod impact value of the composite using unmodified polypropylene increased. But that of composite using maleic anhydride modified polypropylene almost did not change.

(3) Interlaminar shear strength of the composite using maleic anhydride modified polypropylene increased with an increase of crystallinity. Crystallizations of PP and MAPP occurred around carbon fiber after heat treatment. Therefore, interfacial adhesion between fiber and resin increased with an increase of crystallinity.

\section{References}

[1] Information on http://www.teijin.com/rd/technology/cfrp

[2] Fujita, Y., Recent Development of Autemotive Polypropylene, Proc. 2006 Annu. Meet. JSME/MMD (2006), Japan, pp. 483-485 (in Japanese). 
[3] Okada, S. \& Hikosaka, First Direct Observation of Nano-nucleation and Homogeneous Nucleation from the Bulky Melt of Polymer, M. J. Japan. Assoc. Crystal Growth, 37, pp. 34-42, 2010 (in Japanese).

[4] Tang, L. G. \& Kardos, J. L.,A Review of Methods for Improving the Interfacial adhesion between carbon fiber and polymer Matrix, Polymer Composites 18, pp. 100-113, 1997.

[5] Liang J. Z., Quantitative Description of Interfacial strength in Polypropylene/Inorganic Particle Composites, Polymer Composite., 32, pp. 821-828, 2012.

[6] Karsli, N. G. \& Aytac, A., Effect of Maleated Polypropylene on the Morphology, Thermal and Mechanical Properties of Short Carbon Fiber Reinforced Polypropylene Composites, Materials and Design, 32, pp. 4068-4073, 2011.

[7] Mader, E. \& Freitag, K. H., Interface Properties and Their Influence on Short Fibre Composites, Composites, 21, pp. 397-402, 1990.

[8] Fonseca, S. C., Paiva, M.C., Nunes, J. P. \& Bernardo, C. A., A novel technique for interfacial characterization of glass fiber-polypropylene systems, Polymer Testing, 22, pp. 907-913, 2003.

[9] Li, J., The Research on the Interfacial Compatibility of Polypropylene Composite Filled with Surface Treated Carbon Fiber, Applied Surface Science, 255, pp. 8682-8684, 2009.

[10] Varga, J. \& Kocsis, J.K., Interfacial Morphologies in Carbon FiberReinforced Polypropylene Microcomposites, Polymer, 36, pp. 4877-4881, 1995.

[11] Wang, C. \& Liu, C. R., Transcrystallization of Polypropylene Composites: Nucleating Ability of fibres, Polymer, 40, pp. 289-298, 1999. 